\title{
Ein Kochbuch für Tuberkulöse
}

\author{
Cookery Book for Tuberculous Patients
}

\section{Bibliografie}

Dol http://dx.doi.org/

10.1055/s-0029-1244066

Online-Publikation: 13.4. 2010

Pneumologie 2010; 64:

626-631 @ Georg Thieme

Verlag KG Stuttgart · New York

ISSN 0934-8387

\section{Korrespondenzadresse}

Dr. med. Robert Kropp

Liegnitzer Straße 5

36100 Petersberg

dr.robert.kropp@gmx.de
Bis zum Siegeszug der Chemotherapie der Tuberkulose etwa seit der Mitte des letzten Jahrhunderts war das therapeutische Arsenal des Phthisiologen trotz aller Bemühungen zwar umfangreich, aber relativ wirkungslos. Unter den damaligen Behandlungsmöglichkeiten standen die Heilstättenaufenthalte, die Liegekuren sowie die operativen Verfahren, vor allem der Pneumothorax in seinen Varianten, im Vordergrund. Hierzu gehörten auch spezielle diätetische Maßnahmen, eine Therapieform, die recht gut zu vertragen war (vor allem im Vergleich zu den monströsen chirurgischen Verstümmelungen oder zu den „Folterapparaten “ für die Therapie der Knochentuberkulose). Man hatte die Bedeutung der Mangelernährung erkannt. So schreibt E. v. Leyden im Vorwort seines Handbuches der Ernährungstherapie (nach [1]): „Die Diät darf den therapeutischen Anordnungen nicht untergeordnet werden, sie hat ihre eigenen Indikationen und ihre eigenen Leistungen. In manchen Fällen soll sie das allein Maßgebende und allein imstande sein, das Leben zu erhalten und die Gesundheit wieder herzustellen. In solchen Fällen bilden die Medikamente nur eine Unterstützung der Ernährungstherapie.“ Im Folgenden besteht natürlich nicht die Absicht, ehemalige Therapiekonzepte zu empfehlen; vielmehr soll auf die Denkweise früherer Arztgenerationen hingewiesen und ein Blick auf das therapeutische Rüstzeug in der Zeit vor der Chemotherapie $^{1}$ am Beispiel der Diätetik geworfen werden. Seit Jahrtausenden haben Ärzte immer wieder intuitiv versucht, den Kranken durch planmäßige Schonung und Übung unter Verwendung von Licht, Luft und zweckmäßiger Ernährung zu bessern und zu heilen. So spielte die Diätetik seit dem Altertum auch zur Behandlung der Phthise, der Schwindsucht, eine zentrale Rolle. Sehr unter-

\footnotetext{
${ }^{1}$ Um Missverständnissen vorzubeugen: Unter „Chemotherapie“ ist die heutige antituberkulöse Chemotherapie zu verstehen. Die Bezeichnung wurde zuvor auch in anderem Zusammenhang gebraucht.
}

\section{Prïfende Blicke}
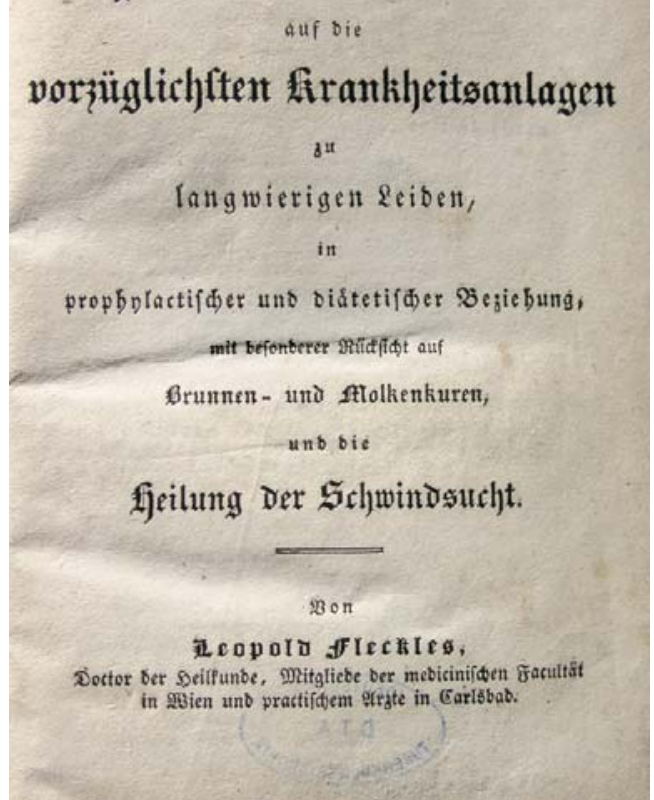

Abb. 1 Titelblatt 1835.

schiedliche Diäten wurden im Laufe der Jahrhunderte empfohlen, nach heutigem Verständnis meist ohne stringente Begründungen, ohne genaue Beschreibung der vermuteten bzw. postulierten Wirkungsmechanismen. Eine ausführlichere Beschreibung ist im Handbuch der Tuberkulose 1923 zu finden [2].

Dies änderte sich erst im Laufe des neunzehnten Jahrhunderts mit der Erarbeitung modernerer wissenschaftlicher Methoden und der genaueren Kenntnis der Krankheit; schon bei einer vergleichenden Betrachtung der beiden Titelblätter ( Abb. 1 u. 2) der Bücher von L. Fleckles 1835 [3] und von G. Rosenfeld [4] 90 Jahre später (1925) meint man, in Schriftbild und Textformu- 


\section{Tuberkulose-Bibliothek}

Beihefte zur Zeitschrift für Tuberkulose herausgegeben von

Prof. Dr. LYDIA RABINOWITSCH

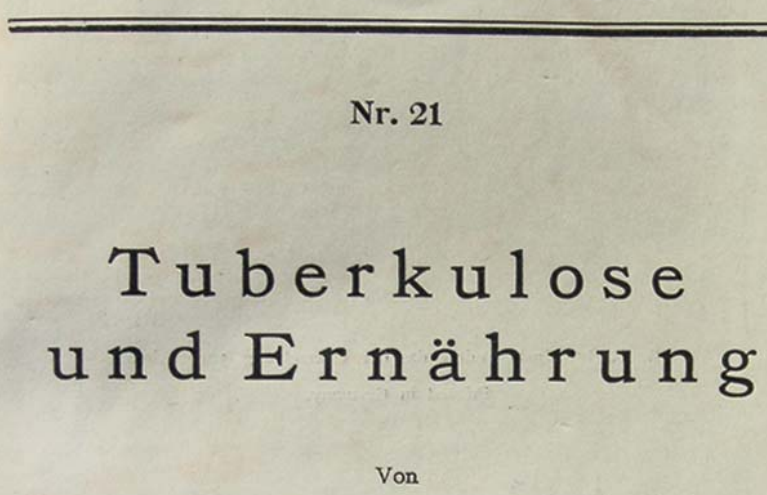

Prof. Dr. Georg Rosenfeld

Geheimem Sanitatsrat in Breslau
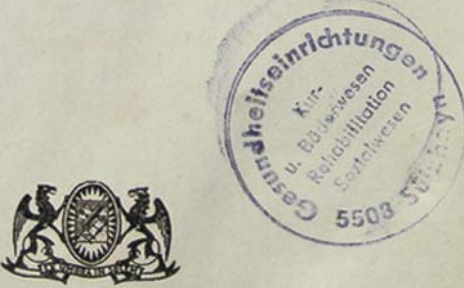

I

$92 \quad 2 \quad 5$

LEIPZIG - VERLAG VON JOHANN AMBROSIUS BARTH

Abb. 2 Titelblatt 1925.

lierung einen Ausdruck dieser unterschiedlichen Auffassungen und Einstellungen zu entdecken.

Hermann Brehmer (1826-1889) war wohl der erste, der eine konsequente Diätetik als essenziellen Teil der Behandlung der Tuberkulose empfahl [5]. Brehmer, der die Heilstätten-Idee entwickelte (seit 1856) und die weltweit erste Tuberkulose-Heilstätte in Görbersdorf/Schlesien gründete, verstand unter einem Heilstättenaufenthalt nicht nur die Isolierung des Tuberkulosepatienten in günstigem Klima; er forderte zusätzlich eine strenge Diät und körperliche Ruhe durch täglich stundenlange Liegekuren. Hierdurch sollten für den kranken Menschen schädliche Einwirkungen in der häuslichen/beruflichen Umgebung (vor allem Unter-, Fehlernährung, miserable Wohnverhältnisse und harte Arbeitsverhältnisse) ausgeschaltet und der Organismus gekräftigt (heute würden wir etwa sagen: die Immunabwehr gestärkt) werden. Brehmers Diätvorschriften bedeuteten eine kalorienreiche Kost, eine „Mastkur“, wie aus einem Beispiel ersehen werden kann:
Speiseplan nach Brehmer für Patienten mit Schluckschwierigkeiten (nach J. Voigt [6], S. 146).

\begin{tabular}{|c|c|}
\hline Morgens $7 \mathrm{Uhr}$ & $\begin{array}{l}250 \mathrm{ccm} \text { Milch mit } 5 \mathrm{ccm} \text { Cognac, dazu eine } \\
\text { geröstete Semmel von } 60 \mathrm{~g}\end{array}$ \\
\hline Morgens $8 \mathrm{Uhr}$ & 1 Tasse Cacao mit Milch gekocht, $180 \mathrm{ccm}$ \\
\hline Morgens $10 \mathrm{Uhr}$ & 1 Glas Milch mit Cognac \\
\hline Mittags & $\begin{array}{l}1 \text { Tasse Fleischbrühe mit Eigelb, } \\
150 \mathrm{~g} \text { Milchreis und } 1 \text { Glas Rothwein }\end{array}$ \\
\hline Nachmittags 4 Uhr & $\begin{array}{l}150 \mathrm{ccm} \text { süßer Milchkaffee mit } 60 \mathrm{~g} \\
\text { gerösteter Semmel }\end{array}$ \\
\hline Nachmittags 6 Uhr & 1 Glas Milch mit Cognac \\
\hline Abends 8 Uhr & $\begin{array}{l}300 \mathrm{ccm} \text { Milchsuppe mit } 60 \mathrm{~g} \text { gerösteter } \\
\text { Semmel }\end{array}$ \\
\hline Nachts & $200 \mathrm{ccm}$ Milch im Glas \\
\hline
\end{tabular}

In den anfänglichen Tuberkulose-Heilstätten wurde von alkoholischen Getränken jeglicher Art reichlich Gebrauch gemacht. Die Auffassungen Brehmers wurden in der Folgezeit verfeinert und modifiziert, zunächst durch seinen Schüler Peter Dettweiler (1837-1904) [7]:

„Die Liegekur bezweckt nichts Geringeres, als durch persönliche Hygiene und Diät, durch stete Belehrung, Überwachung, durch Beispiel und festgeregelte Tagesordnung, nicht am wenigsten durch die Persönlichkeit des Arztes, eine an die jeweilige Leistungsfähigkeit angepasste Lebensweise herbeizuführen bzw. zu erzwingen."

„Wie die Dinge heute liegen, steht die Ernährungstherapie über jeder anderen, sie steht auch in erster Linie bei allen Maßnahmen der Prophylaxe, der Beseitigung der Disposition."

In der Folge wurde die Diätetik ein wesentlicher Teil der Behandlung der Tuberkulosekranken [8,9]. Die folgende Aufstellung zeigt einen üblichen Speisezettel in den Heilstätten zwischen den Weltkriegen [10]:

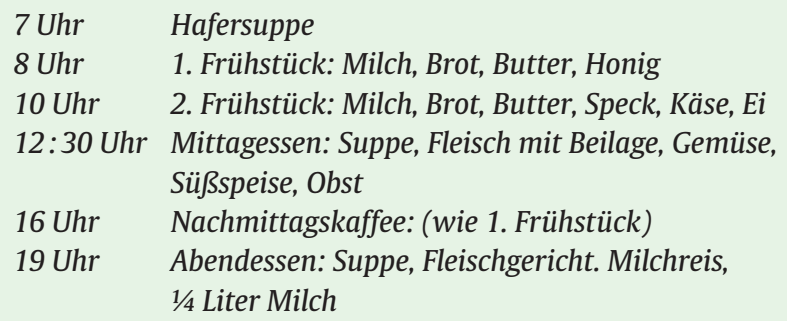

Alkohol war nicht verboten; mäßiger Genuss war unbedenklich. Insbesondere schien es falsch zu sein, ihn Kranken, die ihn lange Zeit regelmäßig zu sich nahmen, von heute auf morgen zu verwehren.

Unterschiedliche Anschauungen über die Art der Kost, der Diät wurden propagiert. Mehrere „Schulen“ entstanden. So sollte die Nahrung vor allem kochsalzarm, -frei sein ${ }^{2}$, „da die Erfahrung gezeigt habe, dass eine kochsalzarme Ernährung für den Verlauf der

\footnotetext{
2 Ausgehend von der Beobachtung, dass Tiere und „Primitivmenschen“ ihre Nahrung kein Kochsalz zur Würzung hinzufügen und (deshalb?) kaum an Tuberkulose erkranken, versuchte man, diese Ernährungssituation zu imitieren, d.h. die Diät für Tuberkulosekranke ohne Zusatz von Kochsalz zu halten $[24,25]$.
} 


\section{KOCHBUCH FÜR TUBERKULÖSE} VON

FRAU MIMICIA HERRMANNSDORFER UND PROF. DR. MED. ET PHIL. ADOLF HERRMANNSDORFER

MIT EINEM GELEITWORT VON

F. SAUERBRUCH

\begin{abstract}
VIERTE ERWEITERTE AUFLAGE DER PRAKTISCHEN ANLEITUNG ZUR KOCHSALZFREIEN ERNÄHRUNG TUBERKULÖSER

MIT 310 MENGENMĨSSIG BERECHNETEN ZUBEREITUNGSVORSCHRTFTEN UND 1 ERNAHRUNGSTABELLE
\end{abstract}

\section{Inhaltsverzeichnis}

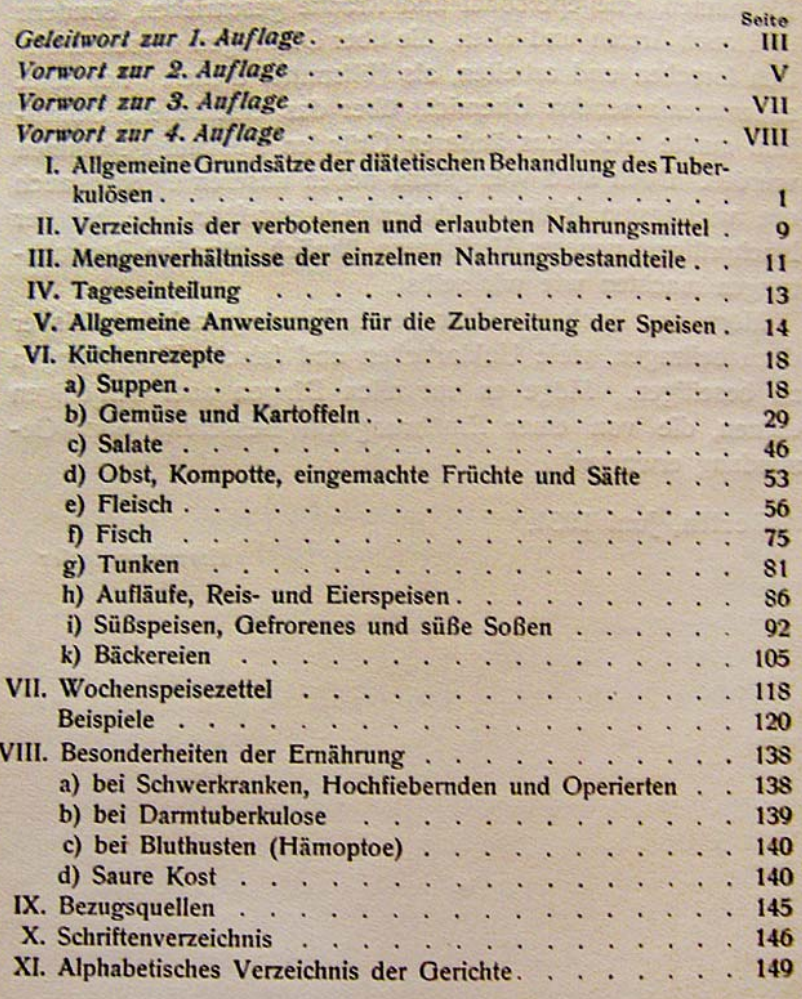

Abb. 4 Kochbuch für Tuberkulöse, Inhaltsverzeichnis.

Die Bedeutung, die Notwendigkeit der Diät bei Tuberkulosekranken wurde allerdings auch bezweifelt $[19,20]$. So schreibt Amrein [21]: „Eine Mästung über das physiologische Maß der Gewichtszunahme bei früher Abgemagerten hinaus ist nicht anzuraten. Sie kann später unliebsame Folgen haben (Herz, Zirkulation usw.). Immer aber möchte ich wieder betonen, dass wir nicht einer verallgemeinernden und alleinseligmachenden Ernährungsform uns verschreiben dürfen; denn eine solche bedeutet nicht eine Notwendigkeit, sondern vielmehr - eine Mode.“

Von der damaligen Bedeutung der Diätetik zeugen auch spezielle „Kochbücher für Tuberkulöse“ (z. B. [22]), deren eines im Besitz des Deutschen Tuberkulose-Archivs ist und hier etwas ausführlicher behandelt werden soll ( Abb. 3). Zweck dieses erstmals 1928 aufgelegten Buches ist ausschließlich die Anleitung zur praktischen Durchführung einer kochsalzlosen, fett-, vitaminund mineralsalzreichen Ernährungsweise, wohingegen hinsichtlich der theoretisch-wissenschaftlichen Grundlagen auf die entsprechende Literatur verwiesen wird ( $\bullet$ Abb. 4).

In einem Eingangskapitel werden zunächst „allgemeine Grundsätze der diätetischen Behandlung des Tuberkulösen“ besprochen. „Gute, kräftigende Ernährung ist eine der wichtigsten Waffen im Kampfe gegen die Tuberkulose in all ihren Erscheinungsformen“ (S. 1), das ist die Devise, deren Verwirklichung allerdings wegen des häufigen Appetitmangels der Patienten große praktische Schwierigkeiten mit sich bringen konnte, auch wegen der grundsätzlich kochsalzfreien Kost. Diese schmackhaft zuzubereiten, war sehr schwierig ${ }^{4}$ und überhaupt nur möglich, wenn „aus-

\footnotetext{
4 „Ohne Kochsalz zu kochen ist eine schwierige Kunst.“ (Seite 14)
}

\footnotetext{
${ }^{3}$ In der Neuauflage des Handbuchs 1958 wird die Diätetik kaum mehr - und wenn, dann vorwiegend unter historischen Gesichtspunkten - behandelt [26].
} überwiegende Überzeugung, „dass die (diätetische [der Autor]) deutung ist. Es darf nicht vorkommen, dass sie den Patienten vo enthalten wird. Es ist auch nicht statthaft, sie in allgemeiner Fasung zu verordnen, sondern sie ist dem einzelnen Kranken streng dosiert anzupassen. Nur so wird der Arzt in der Behandlung der Tuberkulose wirklich künstlerische Arbeit leisten.“ [18] 


\section{Verzeichnis der verbotenen und erlaubten Nahrungsmittel \\ Verbotene Speisen}

Kochsalz.

Konserven jeder Art.

Geräuchertes oder gewürztes Fleisch (Wurst und Schinken).

Geräucherte oder gesalzene Fische.

Bouillonwürfel, Suppenwūrfel und Extrakte, außer den erlaubten.

\section{Erlaubte Speisen}

Salat und Gemüse (roh und gedämpft): Tomaten, gelbe Rüben (Möhren), Schwarzwurzeln, Kohlrabi, Lauch, rote Rüben, Runkelrüben, Teltower Rüben, Spargel, Blumenkohl, Rot- und Weißkraut, gewässertes Sauerkraut, Winterkohl, Rosenkohl, Wirsing, Kresse, Endivien-, Feld- und Kopfsalat, Rhabarber, Sauerampfer, Spinat, Erbsen, Bohnen, Linsen, Pilze, Gurken, Kürbisse, Melonen.

Obst und Früchte: Rohes, aber auch gekochtes, eingeweck. tes und getrocknetes Obst (z. B. Datteln, Feigen, Kastanien, Traubenrosinen, Sultaninen, Nūsse, Mandeln, Dörrobst). Kompotte, Marmeladen, Fruchtgelee, Apfelmost, Mandelmilch. Früchtebrot.

Fette: Salzlose Molkereibutter, Olivenöl, Schmalz (Schweinefett), salzloser Speck.

Milch: Besonders rohe Milch, wenn Quelle einwandfrei; ferner saure Milch, Kefir, Yoghurt, Sahne (Rahm).

Käse: Joghurt-Käse, salzarmer Käse, Quark, Topfenkäse, Parmesankäse (enthält 1,3-1,6\% Kochsalz).

Eier: Auch in Majonnaise, Tunken, Puddings, Cremes, Brei. Frisches Fleisch.

Eingeweide (Milz, Leber, Bries, Hirn, Lunge, Niere).

Frische Fische.

Kartoffeln.

Brot (salzlos): Vollkornbrot, Knāckebrot, Pumpernickel.

Zwieback.

Mehl: Bāckereien, Nudeln, Makkaroni.

Reis (ungeschālter Rangoonreis), Grieß, Maizena, Tapioka, Graupen, Haferilocken.

Zucker: Brauner Kandiszucker, Rohzucker und echter Bienenhonig sind zum Sũßen zu bevorzugen. Schleim. lōsend wirkt Heliosan (bestrahlte Malzhefe), teelöffelweise zwischen den Mahlzeiten zu verabreichen. Der große Vitamingehalt dieses Mittels ist besonders willkommen. Es wirkt ähnlich wie Lebertran.

Bier (,Heilbier", Malzbier, alkoholarmes Starkbier), 3mal wöchentlich 1 Gläschen.

Marsala, Malaga, Madeira, Rotwein und Weißwein, Maraschino, Arrak, Rum (als Zusatz zu den Speisen). Wermutwein (Cinzano), Sekt.

Kaffee, Kakao, Tee (nur in kleinen Mengen als Zusätze zur Milch). Tisane (Deutscher oder Krăutertee, z. B. aus Lindenblüten, Pfefferminz, Kamillen oder dergleichen).

Anmerkung: Die Kost mõglichst trocken halten! Keine Oetrànke auBer den erlaubten!

Abb. 5 Verzeichnis verbotener Speisen.

schließlich sehr zarte und ganz frische Rohstoffe verarbeitet werden. Alle Ersatzmittel sind vollständig unmöglich. “ Im Gegensatz zu anderen empfohlenen Diäten (z. B. [1,9]) beträgt die Zusammensetzung der Diät in diesem Kochbuch:

\section{Eiweiß : Fett : Kohlehydrate $=1,5: 2,7: 4,0$}

Ein Kranker erhielt täglich im Durchschnitt $90 \mathrm{~g}$ Eiweiß, $200 \mathrm{~g}$ Fett (einschließlich $40 \mathrm{~g}$ Lebertran) und $240 \mathrm{~g}$ Kohlehydrate. Es folgt eine Aufzählung verbotener und erlaubter Speisen und Getränke, wobei die Verbotsliste neben Salz stark salzhaltige Nahrungsmittel nennt ( $\bullet$ Abb.5).
Eine ausführliche Gewürz-Liste schließt sich an. „Arzt, Köchin und Pflegepersonal müssen wissen, was und wie viel der Kranke essen soll. Nicht minder wichtig aber ist es, laufend festzustellen, was er nicht isst. Bevor das Geschirr abgeräumt wird, sollte die Diätleiterin auf einem Rundgange durch die Krankenräume sich darüber unterrichten. ... Kranke, die gewohnheitsmäßig schlecht essen oder bestimme Speisen zurückweisen, werden in eine Liste eingetragen, die dem Stationsarzt unterbreitet wird. Diese Maßnahmen tragen wesentlich dazu bei, die Befolgung der Diätvorschriften zu sichern. Eine gut geleitete Ernährungsabteilung hat keine oder nur sehr kleine Abfalleimer."

Anschließend werden allgemeine Anweisungen für die Zubereitung der Speisen gegeben, beginnend mit einer „Tageseinteilung“ der Mahlzeiten ( Abb. 6 ) und gefolgt von einem Kapitel mit 310 (!) Küchenrezepten.

Dieses $^{5}$ enthält 30 verschiedene Suppen (bis zu Hirn-, Leber-, Milz- und Pflaumensuppen), 50 Gemüse- und Kartoffelzubereitungen, 25 Salate, von grünem bis zu Rettichsalat, 10 Kompotte (auch Zuckergurken und gebratene Bananen), 47 Fleischgerichte (etwa auch Minutenfleisch, Pfefferpotthast und Lungenragout!) und 14 Fischzubereitungen. Es folgen Tunken, Aufläufe und Eierspeisen, kalte und warme Süßspeisen (auch „Arrakcreme auf russische Art“ oder „Hasenöhrle“), schließlich Bäckereien. Das Buch schließt mit „Besonderheiten der Ernährung“ bei Darmtuberkulose, bei Bluthusten, für Operierte.

Dieses Kochbuch erlebte mehrere Auflagen und wurde in verschiedene Sprachen übersetzt.

Die einzelnen Rezepte entsprechen im Wesentlichen den hiesigen Koch-Gepflogenheiten. Einige eher ausgefallene oder wohl weniger bekannte mögen zur Illustration dienen ${ }^{6}$ :

Hirnsuppe (Nr. 23). 1 großes Kalbshirn wird gewässert, gehäutet und fein gewiegt. Dann dünstet man es mit $60 \mathrm{~g}$ Zwiebeln und $20 \mathrm{~g}$ grüner Petersilie in $80 \mathrm{~g}$ Butter, stäubt $30 \mathrm{~g}$ Mehl und 1 Teelöffel Nährhefe darüber und gießt mit 21/2 Litern Grundbrühe auf. Dann mengt man 1 Teelöffel Cenovis-Extrakt, 1 Messerspitze Liebig und etwas geriebene Muskatnuss daran und lasse alles aufkochen. Hierauf gibt man gehackte grüne Petersilie daran und legiert mit 2 Eidottern, die man mit 1-2 Teelöffeln Dartex verrührt. Kochzeit: 25 Minuten.

Lungenragout (Nr. 159). 11/2 Pfund Kalbslunge und 1 Kalbsherz kocht man in Grundbrühe mit 1 Zwiebel weich, nimmt das Fleisch heraus und schneidet alles nudelartig. Es wird nun eine braune Schwitze von $80 \mathrm{~g}$ Butter, $40 \mathrm{~g}$ Mehl und 1 Teelöffel Nährhefe bereitet. Diese streut man noch besser erst nach dem Zugießen darüber, weil sie beim Rösten des Mehles leicht bitter wird. Man nimmt die Brühe mit 1 Teelöffel Cenovis-Extrakt und 1/2 Teelöffel Liebig zum Aufgießen, tut die geschnittene Fleischmasse hinein und lässt sie nochmals unter Hinzufügen von 1 Zwiebel, die mit 3 Nelken gespickt ist, mit 2 Lorbeerblättern, etwas Citrovinessig und Zitronensaft, 3 Wacholderbeeren und etwas englischer Soße aufkochen. Kochzeit: 1 Stunde.

Milzklößchen (Nr. 161). $1 \mathrm{~kg}$ Kalbsmilz wird ausgeschabt. Das Mus wird mit 3 Eiern, 3-4 Esslöffeln Mehl und so viel Semmelbröseln vermischt, dass die Masse dicklich wird. Dann gibt man etwas fein gehacktes Basilicum und Zitronensaft daran. Hierauf werden mit Hilfe von 2 Esslöffeln kleine Klößchen in sehr heiße Butter gesetzt und schnell ganz kurz auf beiden Seiten gebraten. Bratzeit: 3-4 Minuten.

\footnotetext{
${ }^{5}$ In Klammern gesetzt sind Beispiele etwas außergewöhnlicher Speisen.

${ }^{6}$ Damalige Produkte wie Cenovis, Dartex, Cinovin u.a. werden nicht eigens erklärt.
} 
7 Uhr Milchsuppe

Danach 1 1/2 Esslöffel Phosphorlebertran (Rp. Phoph. 0,02, Ol. jecor. as. 300,0)

9 Uhr Dünner Kaffee (entweder Malz oder nur wenig Bohnen) mit viel Milch, nach Wunsch auch Milchkakao oder Milchtee; Brot, Butter oder Marmelade oder Honig. Dazu rohes Gemüse (gelbe Rüben, Kohlrabi, weiße Rüben, Blumenkohl, Gurken, Sauerampfer, Sellerie, Rettich, Radieschen, Tomaten, grüne Erbsen (Schoten), frische Maiskolben oder dergleichen).

Diese Rohkost soll im Laufe des Tages verzehrt werden. Bei empfindlichen Verdauungsorganen gibt man statt Rohgemüse eine Tasse Gemüsepresssaft. Schwache und Schwerkranke lasse man auch rohe, mit Zitronensaft beträufelte Eidottern schlucken.

Nach dem Frühstück 1 Teelöffel Mineralogen (in Wasser aufschwemmen und mit Holz- oder Hornlöffel gut verrühren).

12 Uhr Mittagessen: (eine kleine Tasse Suppe), 1 Gang, rohes Obst oder (im Winter) Kompott.

Danach 1 Teelöffel Mineralogen.

3 Uhr Sahne (nach Belieben mit etwas Kaffee oder Tee), Obstkuchen, Keks, Zwieback, Butter- oder Marmalade- oder Honig- oder Früchtebrot oder andere Bäckereien.

51/2 Uhr Abendessen: 1 Gang und Obst.

Danach 1 Teelöffel Mineralogen.

$8 \mathrm{Uhr}$ Milchsuppe (wie morgens); im Sommer statt dessen an heißen Tagen saure Milch.

Danach 1 1/2 Esslöffel Phosphorlebertran.

Kalbsmilch-Auflauf (Nr. 210). 1 kg Kalbsmilch wässert man und entfernt Haut und Blut. Dann tut man sie in Wasser, in dem man 1 Lorbeerblatt, 1 Zwiebel, 1 Nelke, 1 Sträußchen Petersilie, 3-4 Pfefferkörner, 2 Zitronenscheiben, 1 Sträußchen Rosmarin und 2 Esslöffel Weißwein aufgekocht hat. Darin lässt man die Kalbsmilch gar werden, nimmt sie heraus, befreit sie von dem Abfall, überspült sie mit kaltem Wasser und schneidet sie in kleine Würfel. Man bereitet eine holländische Soße, die mit Ei legiert und mit etwas Weißwein und englischer Soße abgeschmeckt wird. Die Kalbsmilch wird nun in eine gebutterte Auflaufform getan. Man gießt die Soße darüber, bestreut alles mit Parmesankäse und belegt mit Butterflöckchen. Dann backt man den Auflauf 10-15 Minuten in heißer Röhre.

Saures Milchgelee (Nr. 237). 1 Liter saure Milch schlägt man mit $120 \mathrm{~g}$ Zucker und dem Saft einer Zitrone glatt. 12 Blatt rote Gelatine weicht man ein, drückt sie aus und löst sie auf; dann gibt man sie an die Milch und schmeckt das Ganze mit 1 Esslöffel Rum oder Arrak ab. Man stellt das Gelee auf Eis. Es kann mit eingezuckerten Erdbeeren gereicht werden.

Dauerkuchen (Nr. 280). 50 g Butter, 200 g Zucker, 3/4 Tasse Milch, 2 ganze Eier, 2 Tassen Mehl, 1/4 Backpulver und eine Handvoll Weinbeeren werden untereinander gemischt, in eine gebutterte Form gefüllt und 3/4 Stunden bei mittlerer Hitze gebacken.

Mit der Durchsetzung der erfolgreichen antituberkulösen Chemotherapie verloren diese ausgefeilten Überlegungen, Vorschläge und Usancen ihren Stellenwert und wurden nur noch gezielt bei manifester Mangelernährung zusätzlich eingesetzt. Denn es konnte nachgewiesen werden, z.B. von der WHO [23], dass der Einfluss der diätetischen Maßnahmen gegenüber der Wirkung der Medikamente zu vernachlässigen ist, etwas, das uns Heuti- gen selbstverständlich, ja banal erscheint und eigentlich keine spezielle Prüfung benötigte.

\section{Interessenkonflikt \\ $\nabla$}

Der Autor gibt an, dass kein Interessenkonflikt besteht.

\section{Literatur}

1 Gerson M. Einiges zur Diätbehandlung der Lungentuberkulose (Rundfunkvortrag vom 18. Februar 1930). Zschr Ärztl Fortbildung 1930; 27 , Nr. 11: 1-13

2 Brauer L, Schröder G, Blumenfeld F. Handbuch der Tuberkulose. 2. Band. Leipzig: Johann Ambrosius Barth, 1923

3 Fleckles L. Prüfende Blicke auf die vorzüglichsten Krankheitsanlagen in prophylaktischer und diätetischer Beziehung, mit besonderer Rücksicht auf Brunnen- und Molkenkuren, und die Heilung der Schwindsucht. Stuttgart: J. Scheible's Buchhandlung, 1835

4 Rosenfeld G. Tuberkulose und Ernährung. Tuberkulose-Bibliothek Nr. 21. Leipzig: Johann Ambrosius Barth, 1925

5 Brehmer H. Die chronische Lungenschwindsucht und die Tuberkulose der Lungen. Berlin: Enslin Verlag, 1857

6 Voigt J. Tuberkulose. Geschichte einer Krankheit. Köln: Vgs Verlagsgesellschaft, 1994

7 Dettweiler P. Die Behandlung der Lungenphthise in geschlossenen Anstalten mit besonderer Beziehung auf Falkenstein i.T. 2. Auflage. Berlin: 1884

8 Zur Diätetik für Lungenkranke. Die Gartenlaube 1868; 16: 39

9 Gerson M. Diättherapie der Lungentuberkulose. Leipzig: Franz Deuticke, 1934

10 Deist H. Die Tuberkulose. Stuttgart: F. Enke, 1951: 229-231

11 Ruhm F. Allgemeine Diätküche. Wien: Ruhm-Verlag, (o.J.) 
12 Bernhardt. Richtlinien für die kochsalzarme Ernährung Tuberkulosekranker (SHG-Diät). Bücher der Hygiene und Volksernährung. Berlin: Rothgießer und Diesing

13 Herrmannsdorfer $M$, Herrmannsdorfer A. Praktische Anleitung zur kochsalzfreien Ernährung Tuberkulöser. 2. Aufl. Leipzig: Johann Ambrosius Barth, 1929

14 Herrmannsdorfer A. Wirkungen kochsalzfreier Ernährung auf tuberkulöse Erkrankungen. Z Tuberk 1930; 56: 257

15 Herrmannsdorfer A. Der Einfluss besonderer Ernährungsart auf schwere Formen tuberkulöser Erkrankungen. Med Klin 1929; 76: 1235

16 Jaffé, Steffens. Hämatologische Untersuchungen bei Hauttuberkulose während der Behandlung mit GHS-Diät. Münch Med Wschr 1929; 76: $703-705$

17 Stamm M. Blutuntersuchungen bei der Behandlung Tuberkulöser mit der Sauerbruch-Herrmannsdorfer-Gersondiät. Z Tuberk 1931; 60: 322

18 Schröder G, Kaufmann K. Ernährung und Kostformen. In: Handbuch der Tuberkulose, 2. Band. Leipzig: Johann Ambrosius Barth, 1923: 100 134
19 Desmo. Nachteile der zwangsweisen Überernährung der Phthisiker. Bullet de thérap 1882; 102: 1

20 Sauerbruch F, Herrmannsdorfer A. Ein klärendes Wort zur ablehnenden Kritik der Ernährungsbehandlung der Tuberkulose. Münch Med Wschr 1930; 77: 43

21 Amrein 0 . Spezielle Ernährungsformen bei Tuberkulose. Mode oder Notwendigkeit? Die Tuberkulose 1930; 10: 115

22 Sternberg $W$. Die Küche in der modernen Heilanstalt. Kochkunst und ärztliche Kunst. Stuttgart: Enke, 1907/09

23 Ramakrishnan CV, Rajendran $K$, Jacob PG et al. The role of diet in the treatment of pulmonary tuberculosis. Bull Org mond Santé 1961; 25: $339-359$

24 Jesionek A. Zur Diätbehandlung der Hauttuberkulose. Münch Med Wschr 1929; 76: 867

25 Jesionek A, Bernhardt L. Diätetische Behandlung der Hauttuberkulose und Ernährungsbiologie. Tuberkulose-Bibliothek (Beihefte zur Zeitschrift für Tuberkulose). Leipzig: Johann Ambrosius Barth, 1930

26 Hein J, Kleinschmidt H, Uehlinger E. Handbuch der Tuberkulose. Stuttgart: Thieme, 1958 